\title{
Early deprivation as a risk factor for narcissistic identity pathologies in adolescence with regard to international adoption
}

\author{
La déprivation affective comme facteur de risque pour les pathologies narcissiques identitaires \\ chez l'adolescent adopté
}

\author{
D. Vandepoel, I. Roskam*, S.-M. Passone, M. Stievenart \\ Psychological sciences research institute, Catholic university of Louvain, 10, place du Cardinal-Mercier, 1348 Louvain-la-Neuve, Belgium
}

\begin{abstract}
The current study is a psychoanalytic reading of the clinical material arising from ongoing developmental research into international adoption within the framework of the Attachment Adoption Research Network (AAARN). The primary objective of the study is to verify whether the severity of deprivation experienced preadoption is a risk factor for narcissistic identity pathologies in adolescence with regard to international adoption. A grounded theory approach is used to identify a set of qualitative variables, which are later quantitatively assessed. The findings are discussed in terms of both qualitative and quantitative results and suggest the greater presence of chronic somatic troubles and observable signs of primary trauma in the adoption population compared to the control group. Future areas for research are suggested in the conclusion.
\end{abstract}

(C) 2014 Elsevier Masson SAS. All rights reserved.

Keywords: Primary trauma; Narcissistic identity pathologies; Somatic trouble; Adolescence; International adoption

\section{Résumé}

La présente étude consiste en une lecture psychanalytique d'un matériel clinique collecté dans le cadre d'une étude développementale portant sur l'adoption internationale: l'Attachment Adoption Research Network (AAARN). Son objectif principal est de déterminer si la sévérité de la déprivation expérimentée avant l'adoption constitue un facteur de risque pour les pathologies narcissiques identitaires à l'adolescence. Une approche théoriquement fondée est utilisée en vue d'identifier un ensemble de variables qualitatives qui seront, dans un deuxième temps, évaluées de manière quantitative. Les résultats sont discutés qualitativement et quantitativement; ils suggèrent la présence accrue de troubles somatiques chroniques, ainsi que de signes observables d'un traumatisme primaire par comparaison avec un groupe d'adolescents contrôle. Des pistes de recherches futures sont suggérées dans la conclusion de cette contribution.

(C) 2014 Elsevier Masson SAS. Tous droits réservés.

Mots clés : Traumatisme primaire ; Pathologies narcissiques identitaires ; Trouble somatique ; Adolescence ; Adoption internationale

\section{Introduction}

It is increasingly accepted nowadays that a primary source of emerging narcissistic identity pathologies is early relational trauma [1-6]. If this is the case, should not severity of deprivation

\footnotetext{
* Corresponding author.

E-mail address: isabelle.roskam@uclouvain.be (I. Roskam).
}

experienced preadoption be a risk factor for narcissistic identity pathologies in adolescence with regard to international adoption?

Despite contrasting empirical research results regarding the long-term effects of international adoption on mental health [7-11], large cohort studies evidence that adolescents and young adults who have been adopted in infancy are overall more at risk of severe mental problems, including suicide or attempted suicide, than their non-adopted peers $[12,13]$. Such higher risk for 
suicide is consistent with the identity disorders often observed in infant psychiatry in the case of adolescents internationally adopted in infancy [14]. What is also clear is that length of preadoption time and severity of caregiving deprivation emerge as two predicting factors of delays in the development of neurological and age-level motor skills, and that the simple enrichment of the adopted child's environment following adoption proves insufficient to repair such damage beyond certain critical ages or sensitive periods $[10,15-18]$. These sensitive periods and cutoff ages differ depending on the institution and country of origin according to the severity of deprivation to which the child has been exposed [19].

International adoption is obviously an extreme early life situation, characterized by intrapsychic and intersubjective discontinuities, all of them potentially traumatic, as well as by exposure to different culture/language (and group symbolic contents) and race/ethnicity. It may therefore represent a limiting case in the mathematical sense, to which it is worth applying theoretical concepts regarding the effect of trauma on early cognitive and emotional development. The current research draws on two psychoanalytical models regarding the effect of trauma on early cognitive and emotional development: Bion's alpha function and Roussillon's primary trauma. Their main concepts are shortly reviewed here, together with their consistency and reliability in regard to the limiting case of international adoption, in the light of the findings of recent neurobiological research on early relational trauma.

\subsection{Alpha function and somatization}

Both attachment theory and psychoanalytical models agree on the essential role played by the caregiver's response to the child's expression of need [20]. Bion considered the endogenous and exogenous perceptions of a young child in distress as preliminary forms of thought resulting from an uncompleted symbolization process. The malleability of the object's response expressed by Bion's concept of maternal reverie ensures, on the part of the child, the alpha function that transforms perceptions into first representations, enabling the completion of primary symbolization. It is a key requisite for a seamless transition to the secondary symbolization involved in the affective and cognitive regulating functions of the self [21-23]. The child progressively internalizes and stabilizes in contact with his or her primary caregivers, the alpha function, prompting the organization of the self through the prism of a contact barrier constructed from alpha elements designed to buffer contrasting experiences of the primary object's level of attention (absence/presence, satisfaction/frustration, good/bad experiences) and leading to the integration of ambivalence. As a result, it becomes possible for the child to overcome the depressive position in the sense of Klein [24] which occurs mainly in the second six months of life with the disillusion that follows the loss of infant fantasies of omnipotent control, but which is revisited throughout a person's life whenever a loss is experienced [25]. Conversely, the experience of a child who is dramatically and/or long-lastingly deprived of maternal reverie, and hence, unable to transform perceptive traces into a symbolized material, is one of terror [22], and stabilization of the alpha function is compromised. Later in the course of development, the subject deprived of a stabilized alpha function is also more at risk of unloading excessive excitation back onto the soma. The immune system will have difficulty coping with this load, and this paves the way for chronic somatic troubles. The ages at adoption of the adolescents studied here and the defining of these age groups are therefore expected to be a first critical design consideration in order to assess the alpha function degree of internalizing in adolescence.

\subsection{Primary trauma and state of agony}

Roussillon [26] offers in the same line of influence as Bion a psychoanalytical model in three stages which includes dimensions of caregiving deprivation severity and duration as a means of describing early and ultra-early traumas associated with the terror experience generated by the failure of the maternal reverie. The model describes how a situation that is only potentially traumatic becomes increasingly traumatic depending on how the environment responds to the child's distress. Failure at the first stage, in which the child attempts to draw upon internal mental resources to bind or discharge the influx of excitations leads to a second stage characterized by helplessness, in which the child tries to set up a narcissistic contract with the primary object in order to reduce anxiety. The contract is narcissistic because it is tainted by the caregiver's insufficiently malleable response, and involves a price that the child has to pay. If setting up a narcissistic contract fails because the object's response is too unsatisfactory or because the price to pay is too high, the overwhelming excitation breaks through the child's protective shield, exerting a mental violence that mobilizes an impotent rage and further exacerbates the state of helplessness. The child tries to get rid of this rage by projecting it on the object. This violence is correlated with a sense of primary guilt, very distinct from the secondary guilt associated with overcoming the Kleinian depressive position. The third stage of the model happens when the state of helplessness becomes unbearable in its duration and escalates the primary traumatic situation into a state of agony that can produce the terror theorized by Bion [23]. This traumatic state beyond helplessness and hope (state of agony) induces an existential despair. According to Roussillon, the subject prefers to feel guilty and responsible and therefore in control for having failed to cope with what he or she was faced with, rather than facing the sense of helplessness associated with the agonizing experience. Thus, the subject fights to maintain the illusion of omnipotent control, instead of integrating the aspects of ambivalence.

\subsection{Splitting and narcissistic identity pathologies}

In contrast with the objectivist approach that dominates psychiatric classifications of narcissistic disturbances, Roussillon's theoretical approach provides a comprehensive approach to psychological pain, by considering the symptom as an expression of a mental disorder determined by types of anxieties, defense mechanisms and object relationships. Roussillon theorizes that in order to survive the state of agony, the child withdraws from 
Table 1

Main and additional defense mechanisms associated with primary trauma.

\begin{tabular}{|c|c|}
\hline Organization of defense mechanisms & Consequences \\
\hline $\begin{array}{l}\text { Main defense mechanism against experience of primary agony (primary trauma): } \\
\text { Splitting }\end{array}$ & $\begin{array}{l}\text { Compulsion to repeat/split off tends to return to and } \\
\text { reproduce the traumatic state itself }\end{array}$ \\
\hline $\begin{array}{l}\text { Additional defense mechanisms to counter the return of split off trauma } \\
\text { Amputation of part of the self to maintain a relation with the object and regress from } \\
\text { state of agony to state of helplessness } \\
\text { False Self (part of the mental system is dedicated to protecting the (true) self against a } \\
\text { world felt to be unsafe) } \\
\text { Global organization of the mental life that seeks to limit object investment and in } \\
\text { general all relationships that can evoke the primary trauma area and its state of } \\
\text { helplessness }\end{array}$ & $\begin{array}{l}\text { Impoverishes the self and therefore leads to narcissistic } \\
\text { identity pathologies; } \\
\text { Unrepresented part of the mental system generates } \\
\text { excitation in excess }\end{array}$ \\
\hline $\begin{array}{l}\text { Others solutions aimed at binding excessive excitation due to unrepresented experiences } \\
\text { Tie thanks to sexual excitation } \\
\text { Absorb by placing excessive emphasis on perceptions and sensations } \\
\text { Try to symbolize secondarily what was not successfully symbolized at the primary } \\
\text { stage using delirium (or the "return" of the perceptive memory trace) }\end{array}$ & \\
\hline
\end{tabular}

the primary traumatic experience in order to stop feeling, and this withdrawal "beyond the self" is described as a form of splitting. The paradox is that the self splits from an experience that was felt and that therefore produced lasting memory traces but did not gain a representation. In the course of the primary symbolization process which transforms perceptions into first representations, a failure occurs that prevents its fulfilment and thereby, also inhibits the further processing of secondary symbolization. Roussillon makes the assumption that the primary trauma that has been split off is governed by the compulsion to repeat and tends to return to and reproduce the traumatic state itself. Additional mechanisms appear beside the principal mechanism of splitting in order to prevent the return of the traumatic state, and in this way, an improvised solution is structured into a defensive syndrome. Table 1 provides an overview of the main and additional defense mechanisms involved and how those impoverish the self and therefore lead to narcissistic identity pathologies, the unrepresented part of the mental system generating excitation in excess. Roussillon regards splitting as the key mechanism behind all forms of narcissistic identity pathologies, while the defensive syndrome used to prevent the return of the split off primary trauma defines the form of the disorder [27]. Two clinical illustrations of how splitting and defense against the return of the split off trauma might operate with adoptees are given in the work of Fagan [28] and Harf et al. [29]. Such a model draws a theoretical link between primary trauma, primary violence and guilt, antisocial behavior and somatic and identity disorders. It is supported by recent neurobiological research [30-33]. It is also consistent with the recurrent comorbidity observed in infant psychiatry between somatization and psychopathic personality traits [34].

\subsubsection{Adoption as an amplifier of fantasies in adolescence}

Adolescence is a sensitive period where alpha dysfunction may be revealed even more starkly, as puberty involves major neural changes in the affective and executive regions of the brain [35-37], and adolescents detaching from their primary objects have to rely increasingly on their own resources, leaving their narcissistic vulnerability exposed [38]. Such reorganizations are likely to reactivate potential infantile elements which were still pending secondary symbolization (e.g. fantasies) on the part not only of adolescents but also of their parents. Adoption thus plays a role as an amplifier of the cognitive and affective reorganizations specific to puberty, as adolescents' and parents' fantasies resonate with each other. Typical fantasies related to parentage and the search for origins in adolescence are more complex for adoptees and a fortiori for those who were internationally adopted than for their non-adopted peers, as the former deal with intrapsychic representations of both adoptive and biological parents, who are often characterized by opposite valences. The effects of puberty and chronological age are not closely correlated in early adolescence, especially with regard to affective brain development [39]. This highlights the difficulty of categorizing and defining age groups appropriately, and for the current study, the ages of the participants studied and the labels used for different age groups are expected to be another critical design consideration.

\subsection{Objective and addressed question}

The question addressed in this study was "Is severity of deprivation experienced preadoption a risk factor for narcissistic identity pathologies in adolescence with regard to international adoption?" In order to address this question, a grounded theory approach [40] was used to analyze the transcripts of semi-structured interviews conducted with 50 adolescents and their parents according to proven and validated variants of the AAI protocol, the Parental Development Interview [41] and Friends and Family Interview [42] respectively. Potentially suitable themes and categories emerging during coding were classified firstly as types of anxiety, defense mechanism and object relationship, secondly according to degree of alpha functioning and finally in relation to forms of narcissistic identity pathologies according to Roussillon's classifications. All 
subjects - both adolescents and parents - also self-administered the Child Behaviour Check List [43].

\section{Method}

\subsection{Participants}

The original developmental research framed by the Attachment Adoption Adolescents Research Network (AAARN) included 80 Belgian adolescents and their parents, recruited using a convenience sampling approach, with the study being advertised in the local area.

Cases for which data were missing, partial or extreme were discarded here. As a result, the current research focuses on a core group of fifty adolescents (25 girls) aged 10 to 16 , of whom 25 had been adopted internationally in infancy or in childhood, and of their mothers and fathers. The adolescents were raised in families with mid-to-high socioeconomic status, i.e. the parents' education level was moderate or high and they had access to the labor market.

Table 2 summarizes the participants' distribution by gender, age group at the time of interview, age group at the time of adoption, region of origin and adoption family siblings. The age group design underlying this distribution is based on critical guidelines discussed in the introduction. CBCL data were available for all subjects. An additional group of 11 adopted adolescents initially discarded from the study (e.g. because the father's interview was missing, or because the adoption was national instead of international) participated in the final testing of the model developed during the research (see also Table 2), in order to test and validate the limits of the model.

\subsection{Interviews and transcripts}

The Parent Development Interview (PDI) is a 59-item semistructured clinical interviews designed to examine parents' representations of their children, themselves as parents, their relationships with their children and their past relationships with their own parents. The Friends and Family Interview (FFI) is a 36-item semi-structured clinical interview designed to examine adolescents' representations of self, friends and family. Both are designed to assess internal working models of relationships and are an autobiographical memory measure [42]. Interviews were conducted by pairs of extensively trained master's students in developmental psychology, according to clearly defined protocols $[44,45]$. The Child Behavior Checklist [43] is a widely used parent and child-report measure of emotional and behavioral problems in young people from both clinical and research perspectives. The derived six CBCL DSMOriented Scales were constructed through agreement in ratings among 22 highly experienced child psychiatrists and psychologists from 16 cultures.

Table 2

Participants (adolescents).

\begin{tabular}{|c|c|c|c|c|}
\hline \multirow[t]{2}{*}{ Group categories } & & \multicolumn{3}{|l|}{$n(\%)$} \\
\hline & & Core adoption group & Large group (Core plus Additional adoption group) & Control group \\
\hline Mothers & & 25 & 35 & 25 \\
\hline Boys & & $12(48 \%)$ & 18 & $14(56 \%)$ \\
\hline Girls & & $13(52 \%)$ & 18 & $11(44 \%)$ \\
\hline $13-14 y$ & & $7(28 \%)$ & 8 & $7(28 \%)$ \\
\hline $15-16 y$ & & $7(28 \%)$ & 12 & $9(36 \%)$ \\
\hline \multicolumn{5}{|l|}{ Age range at adoption } \\
\hline First semester & $0-5$ months & $6(24 \%)$ & 10 & - \\
\hline Second six months & 6-11 months & $9(36 \%)$ & 10 & - \\
\hline Second year & 12-24 months & $5(20 \%)$ & 7 & - \\
\hline Eastern Europe & & $1(5 \%)$ & 3 & - \\
\hline South America & & $8(32 \%)$ & 12 & - \\
\hline Africa & & $4(16 \%)$ & 5 & - \\
\hline National & & - & 2 & - \\
\hline \multicolumn{5}{|l|}{ Family configuration } \\
\hline Adopted sibling only & & $14(56 \%)$ & 22 & - \\
\hline Biological sibling as well & & $11(44 \%)$ & 14 & - \\
\hline Biological sibling only & & - & - & $25(100 \%)$ \\
\hline Total & & 25 & 36 & 25 \\
\hline
\end{tabular}


Table 3

Data analysis plan.

\begin{tabular}{|c|c|c|}
\hline \multicolumn{3}{|l|}{ Analysis plan } \\
\hline Thematic analysis & $\begin{array}{l}\text { Stage-by-stage } \\
\text { process }\end{array}$ & Results \\
\hline Deductive reasoning & Open coding, & Final themes, \\
\hline Theoretical sampling & Axial coding, & categories and \\
\hline Single cases oriented approach & Elective coding & their dimensions \\
\hline Inductive reasoning & & and properties \\
\hline Primary trauma model & & \\
\hline Narcissistic identity pathologies & & \\
\hline Variable oriented approach & & \\
\hline Quantitative approach & \multicolumn{2}{|c|}{ Validation - Comparison } \\
\hline
\end{tabular}

\subsection{Data analysis}

Table 3 gives an overview of the methodological backbone that sustained the analysis. The data analysis process was non-linear, combining qualitative and quantitative procedures, deductive and inductive reasoning, single cases and variable oriented approaches. The framework of psychoanalytical theory always prevailed and is a primary element of the researcher's subjectivity. From a chronological point of view, the first phase was purely qualitative, using thematic analysis, a suitable method for exploring the large data set. The process of data analysis oscillated back and forth between data-driven and theory-driven approaches; the emerging theoretical hypothesis of primary trauma anticipated in the theoretical introduction of this paper and its verifications were held in constant interaction until the saturation of categories [46]. The final theoretical items were limited to the themes and categories validated by the data. The final results regarding adolescents can be found in Table 4. The development of reports emerged from the detailed coding tables and summarizes their outcome; meanwhile, underlying ideas were recorded in memos. The quantitative phase converted a selective set of categories into dichotomized variables, computed them into SPSS 21 and compared their values to the available CBCL DSM-oriented Scales scores through one way ANOVA treatment, the $\mathrm{Chi}^{2}$ test and Pearson correlations.

\subsubsection{Thematic analysis and measurement tools}

As summarized in Table 3, the stage-by-stage data analysis process included open coding, axial coding and selective coding. It started with the reading of a theoretically sampled set of cases in the adoption group and in the control group in order to saturate group categories using a single case approach; it was completed after testing the resulting model with the enlarged group of cases, including the additional data set of eleven cases, using a variable oriented approach. Emerging themes and categories and recurring discursive structures drove the design of generations of coding tables embedding the individual affective and cognitive organizations identified for each adolescent and parent.

\subsubsection{Measures}

During open coding, text fragments from parents and adolescent's transcripts were cross-tabbed according to their properties and dimensions (e.g. type of affect, representation, conflict, defense mechanism, object relationship) in first-generation coding tables using a data-driven approach. The coding table, which was theoretically anchored at the beginning of the analysis, became simplified and focused once recurrent elements had appeared. During axial coding, the search for repeated intergroup patterns led to the identification of distinct categories (e.g. projection as defense mechanism, dual relationship). Selective coding was articulated around the research question in regard to narcissistic identity pathologies as an outcome of early trauma, due to severity of caregiving deprivation, firstly roughly estimated by age at adoption [19] and then by group. Key categories which could embed groups of categories were defined. Insufficiently represented categories were discarded.

A coding journal and coding memo were created in order to ensure internal reliability. Coder reliability was established in two ways:

- during open coding, three interviews in both transcript and video recording form were submitted to a group of clinicians working in the childcare sector. Those experts came out in an independent group session with all the categories identified by the researcher in terms of mental mechanisms and discovered no additional ones;

- during selective coding, emerging key categories and their sub-hierarchy were later discussed and reviewed with experts in narcissistic identity disorder and infant psychopathology.

During axial coding, triangulation was achieved by collecting and cross-tabulating data from both parents and adolescents. In order to establish the external validity of the qualitative method, the findings regarding one key category were quantitatively compared with those provided by the CBCL data collection. The discussion creates a dialogue between qualitative and quantitative results.

\section{Results}

\subsection{Thematic analysis}

Table 4 reports the final categories of anxiety and defense mechanisms that emerged from the triangulated analysis of parents' and adolescents' interview transcripts (deriving from the data-driven approach applied during open and axial coding). Their occurrence (overall) and frequency (number of adopted adolescents for whom the category was spotted at least once) are quantified for both the core $(n=25)$ and the large $(n=36)$ groups of adoptees. Categories are hierarchically organized according to the research question following the model proposed by Roussillon [26] (theory-driven approach) as signaling the existence of primary trauma correlated to later narcissistic identity disorder (e.g. amputation of a part of the self: refusal to think or learn about origins), defense breakdown (e.g. return of split off trauma: state of agony triggered by cyclic events) and other solutions aimed at binding excessive excitation (e.g. exaggerated emphasis on perceptions or sensations: the child can only associate the experience of separation with smells). The 
Table 4

Report on the thematic analysis for adolescents adopted in infancy or childhood.

\begin{tabular}{|c|c|c|c|c|c|c|c|}
\hline \multirow{2}{*}{$\frac{\text { Key category }}{n^{*}}$} & \multirow[t]{2}{*}{ Category } & \multicolumn{2}{|c|}{ Number of codes } & \multicolumn{2}{|c|}{ Number of adolescents } & \multicolumn{2}{|c|}{$\%$ adolescents } \\
\hline & & 25 & 36 & 25 & 36 & 25 & 36 \\
\hline \multicolumn{8}{|c|}{ Signs of primary } \\
\hline \multirow[t]{5}{*}{ Trauma } & Helplessness & 24 & 36 & 16 & 25 & 64 & 69 \\
\hline & Shame & 10 & 13 & 8 & 10 & 32 & 28 \\
\hline & State of agony when sick & 10 & 14 & 10 & 15 & 40 & 42 \\
\hline & Control & 22 & 32 & 14 & 21 & 56 & 58 \\
\hline & Hyper-excitation & 16 & 22 & 13 & 18 & 52 & 50 \\
\hline Somatizing & Chronic troubles & 17 & 25 & 13 & 19 & 52 & 53 \\
\hline \multicolumn{8}{|l|}{ Alpha function } \\
\hline & Functioning & 23 & 30 & 14 & 18 & 56 & 50 \\
\hline & Dysfunction & 42 & 57 & 18 & 24 & 72 & 67 \\
\hline \multicolumn{8}{|c|}{ Others solutions } \\
\hline
\end{tabular}

* Core group: $n=25$; Enlarged group (core + additional set): $n=36$.

mapping schema between categories and narcissistic defenses is illustrated in Table 5.

\subsubsection{Defense mechanisms among adopted adolescents}

The content of information appeared at the outset to differ substantially according to the age of the adolescent at the moment of interview. The sharp anxieties and defense mechanisms were self-describing in the group of 10-12 years old, while false self and strategies for negotiating with authority appeared more clearly in the older groups.

Overall however, adoptees seemed more likely to develop extreme defense mechanisms than their non-adopted peers, displaying:

- strategies of control (56\%), mainly in order to preserve freedom or to manage change, as all transitions and separations are difficult and associated with fear/stress;

- compliance (64\%), e.g. over-attentiveness to others' mental state, trying to agree, psychological insight, acuity, finesse;

- withdrawal (68\%), e.g. avoiding contact when feeling sad, self-representation as lazy;
- feelings of helplessness (64\%), e.g. triggered by threat of disruption in object relationship due to conflict;

- episodes of rage (56\%) triggered by feeling of injustice, e.g. a loss was hidden from the child, with projection and denial of feelings of anger (and fear).

Although helplessness is often denied, either by the adolescent (e.g. denial of loss) or by the parents (e.g. trivializing daughter's needs for attention), many adoptees, once overwhelmed, withdraw out of reach in what parents described as their own fantasy world. A remarkable type of object relationship seemed to subsist during this withdrawal, as the child or preadolescent in distress often first sought contact with a pet. Half of the adopted adolescents in the 10-12-year-old group and $36 \%$ overall referred to a special relationship with a familiar cat, rabbit or horse.

The collapse of defenses often appeared in correlation with the return of the state of agony (e.g. triggered by cyclic events: birthdays, family celebrations or by reliving the experience of loss: losing a friend, associated with fantasy of death) and could take the form of somatization (e.g. feeling like dying when sick).

Table 5

Narcissistic defense syndromes and category mapping.

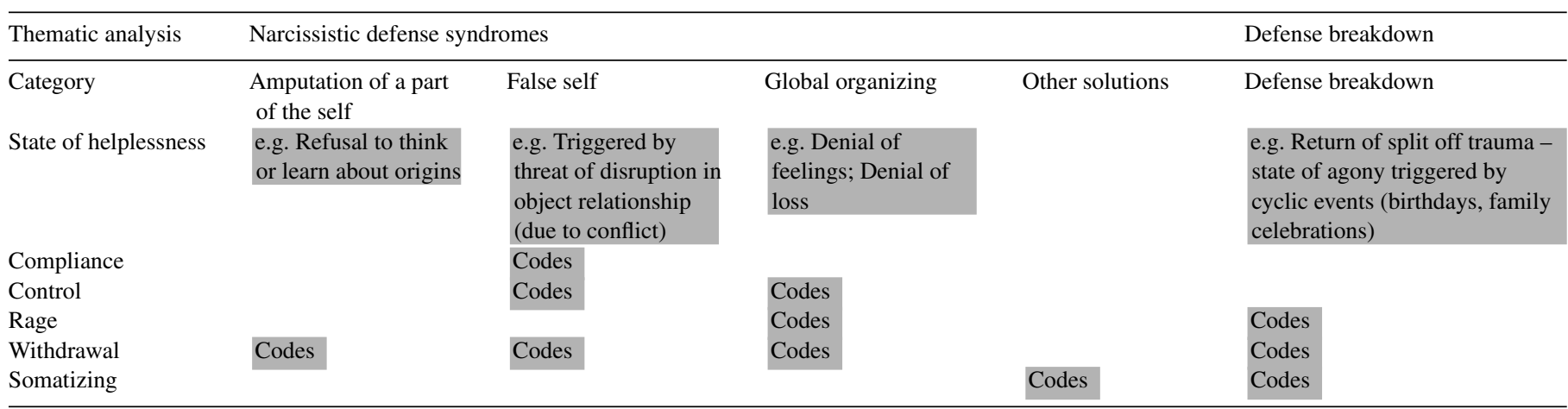


The input provided by the analysis of the 10- to 12-year-old and their parents evokes the existence of the split off part of the adolescent's self that suddenly nears the surface, giving the parents the strange feeling that the child or the adolescent is at the same time there and not there and "actualizing the stupor - the archaic terror" - that we can assume the child once experienced and that now threatens the relationship. Suicide attempts or risks were mentioned by the parents for 3 adoptees and 1 biological child. Only in the case of the biological child did the mother associate the suicide attempt with her own brother's suicide. This frequency, despite being statistically insignificant with this small size sample, matches the risk ratio obtained by Hjern and al. [12].

\subsubsection{Other observed solutions aimed at binding or discharging excessive excitation}

The other observed solutions are:

- contrast between physical maturity (e.g. early sexual activity as an attempt to integrate traumatic experiences which have not been worked through by means of sexual excitation) and affective immaturity, with a delay in affective and cognitive mentalizing (e.g. adoptees reported to be immature in terms of understanding and hence also learning: they need to "replay" dialogs in order to grasp emotions or receive attention from others in order to learn concepts);

- sensory perceptions are a first means of describing experiences of separation while memories are still missing. Sounds and music play a role in providing an experience of accordance with others but also help with thinking and conceptualizing, calm anxiety or open up a transitional space for encounter with parents and peers [47];

- the sense of belonging offered by the group of peers was another solution frequently used by the adoptees (in youth clubs and sports teams).

\subsubsection{Alpha function}

Ten to 12-year-old adoptees tended more than their nonadopted peers to have developed only one side of the ambivalence. Feelings of sadness and depressive feelings are often mentioned which recall here the early deprivation correlated with the primary state of agony experiences rather than the overcoming of the depressive position. Among adoptees, it seemed more frequent for the ambivalence (normally resolved by the overcoming of the Kleinian depressive position) to extend largely beyond normal age. It appears only to be resolved later, within the 15-16-year-old group, who seemed to integrate contrasting emotions. Adolescents of 10-12 and 13-14 years of age were observed to more frequently verbalize how they struggled with a dysfunction of the alpha function with issues of absence/presence (e.g. excessive attention to others' presence), temporality when faced with uncertainty, which induces stress and impatience (e.g. time is necessary to handle constraints, planning is an issue) and transitionality (e.g. "all or nothing" attitude associated with sleep problems and hyper-excitation; lack of access to feelings, or difficulty with expressing feelings). As said above, calming anxiety (which can lead to the internalizing of the alpha function) often seemed to be mediated by a close relationship with a pet.

In order to verify whether the categories were more specific to adoptees than to non-adopted adolescents, they were quantified and systematically measured in the adoption and control groups, where applicable in relation with group design variables (age at adoption and age at interview) in order to rebut or confirm the assumptions that emerged from the thematic analysis. Two key categories, signs of primary trauma (embedding the extreme defense mechanisms observed and presumably put in place to prevent the return of the split off primary trauma) and somatic troubles (correlated with chronic/genetic disease, and or associated with early infancy illness) were computed in SPSS 21. One way ANOVA analysis and $\mathrm{Chi}^{2}$ tests were performed and verified for:

- sample independence, equality of variances and equality of means for the first;

- sample independence and expected count for the second.

\subsection{Quantitative analysis}

\subsubsection{Variables}

Categories taken into account as signs of primary trauma were rage, excessive control -in its preventive and anticipating function, compliance, withdrawal when upset or sad, hyperexcitation and feeling a state of agony when sick. The result is a qualitative dichotomous variable assessed on the basis of the triangulated interviews, with at least two main signs of primary trauma needing to be identified for that adolescent. Somatic troubles is a dichotomous variable assessed by triangulating data from the interviews of the adolescent and both parents. The criteria of inclusion or exclusion were based on the chronic nature of the troubles [48], which were divided into three nonexclusive categories:

- early somatic troubles in infancy;

- genetic disease;

- chronic somatic troubles in childhood and/or adolescence.

The qualitative indicator was next adjusted after co-varying out cases with genetically sourced or early infancy diseases that pre- and postnatal malnutrition and poor healthcare could explain. The adjusted indicator was therefore a more likely measure of the existence of chronic somatic troubles generated by mental shortcomings due to the problems with the primary symbolization process. Finally, an unusual parameter was also measured, namely whether a relationship with a pet was important to the child/adolescent.

CBCL DSM-oriented scales constructed from the CBCL collected data assessed by adolescents and parents were also taken into account in the search for possible correlations. The scales considered were the Somatic Problems Scale and Conduct Problems Scale [49].

The independent variables taken into consideration were the group [adoption, control], the age at adoption in four ranges 
Table 6

Comparison between adoption and control group $(p<0.05)$.

\begin{tabular}{|c|c|c|c|}
\hline \multirow[t]{2}{*}{ Categories } & \multicolumn{2}{|l|}{$n(\%)$} & \multirow[t]{2}{*}{$p$} \\
\hline & Adoption group $n=25$ & Control group $n=25$ & \\
\hline $\begin{array}{l}\text { Signs of primary trauma (at least two of following criteria filled: } \\
\quad \text { rage, control, compliance, withdrawal, state of agony when sick }\end{array}$ & $19(76 \%)$ & $12(48 \%)$ & 0.04 \\
\hline Somatic troubles & $17(68 \%)$ & $11(44 \%)$ & 0.04 \\
\hline Chronic & $13(52 \%)$ & $6(24 \%)$ & \\
\hline Early & $8(35 \%)$ & $6(24 \%)$ & \\
\hline Genetic & $3(12 \%)$ & $2(8 \%)$ & \\
\hline Somatic troubles (adjusted) & $9(36 \%)$ & $2(8 \%)$ & 0.02 \\
\hline Attachment to pet & $9(36 \%)$ & $2(8 \%)$ & 0.019 \\
\hline CBCL DSM-oriented Somatic Problems Scales & Average score (SD) & & \\
\hline Parents & 0.78 & 0.25 & 0.036 \\
\hline Adolescents & 2.7 & 1.0 & 0.034 \\
\hline
\end{tabular}

[0-5 months, 6-11 months, 12-24 months, over 24 months] and the age at time of interview in three ranges [10-12 y, 13-14 y, 15-16y). The four ranges of age at adoption were defined in the light of the sensitive periods identified in early cognitive and affective development [25] and correlate to the age groups defined in the original developmental research [44]. Empirical research identified a cut-off age of 6 months at adoption for no neurological delay compared with non-adoptees, and the second age range of 6-11 months was particularly designed to observe the degree of internalizing of the alpha function. A fifth group was initially added that took into consideration the experience of children adopted during their first three months of life. Although this categorization provided interesting results, it was biased as adding a group resulted in less significant samples in the other group. The three ranges finally defined to assess the effect of age at interview consider the input of neurobiological research regarding the impact of puberty on affective functions development in the brain, with two extreme age groups and a group centered on the critical 14-15 years period. See Table 2 for the number of participants in each of these categories.

\subsubsection{Main adoption effects}

Table 6 shows that although signs of primary trauma were also present among peers within the control group, they were significantly more frequently observed in the adoption group $\left(76 \%\right.$ vs. $\left.48 \% ; \chi^{2}=4.16 ; p=0.040^{1}\right)$. Somatic troubles were also significantly more frequent in the adoption group $(68 \%$ for adoptees vs. $44 \%$ for non-adopted; $\chi^{2}=4.16^{1}, p=0.04$ and $36 \%$ vs. $8 \% ; \chi^{2}=5.71^{2}, p=0.02$ after adjustment). They included chronic digestive disorders induced by stress (diarrhea, stomach ache), chronic sleep problems with anxiety at the moment of

\footnotetext{
1 Zero cells $(0.0 \%)$ have expected count less than 5 . The minimum expected count is 9.5 .

2 Zero cells $(0.0 \%)$ have expected count less than 5 . The minimum expected count is 5.5 .
}

going to sleep or at night, and recurrent episodes of bronchitis, pneumonia, allergies, eczema and asthma.

\subsubsection{Correlation between Somatic Problems Scale and somatic trouble (adjusted)}

The CBCL DSM-oriented Somatic Problems Scale measured with parents and with adolescents, quantitatively scored, could add a severity indication to the somatic troubles observed in the two groups. Results are summarized in Table 6. Both parents' and adolescents' assessment scores showed on average a significant adoption effect $(F=4.68 ; p=0.036$ and $F=4.808$; $p=0.034$ respectively), values being on the pathological side when assessed in adoptees. Moreover, somatic problems and somatic trouble correlate moderately overall $(r=0.52 ; p=0.000$ for parents and $r=0.31 ; p=0.038$ for adolescents), which makes sense since most qualitative information regarding the adolescents' somatic troubles was provided by analysis of the parents' transcripts.

\subsubsection{Correlation between somatic problems and antisocial personality disorder}

The association between antisocial personality disorder and somatization disorder is a co-morbidity consistently reported in the psychopathology literature, confirming the idea of a shared etiological origin [34]. It is also an idea already suggested in the review of the psychoanalytical literature, associated with a poorly internalized and/or stabilized alpha function and the construction of the contact barrier. Lilienfeld and Hess [34] found that only secondary psychopathology - the antisocial lifestyle component of psychopathy rather than the core affective traits - correlates with somatization disorder. Other DSM-Oriented Scales constructed from the CBCL collected data were also analyzed using ANOVA I. The only other adoption effect observed was that adoptive parents rate conduct problems higher than biological parents do $(F=5.9 ; p=0.019)$ and higher than their children do. Actually, the somatic problems score assessed by parents correlates overall with the conduct problems assessed by them $(r=0.55 ; p=0.000)$, a correlation 
that remains significant after ages and gender variables have been controlled for.

\subsubsection{Pet attachment}

As indicated in Table 6, adopted children are indeed more likely to attach importance to a relationship with their pet $\left(\chi^{2}=5.71, p=0.019^{3}\right)$, especially in childhood and preadolescence.

In summary, the quantitative analysis suggests the greater presence of observable signs of primary trauma and chronic somatic troubles in the adoption population compared to the control group. The difference in average between adoption and control groups is reflected by the DSM-Oriented Scale scored by parents and adolescents for the somatic troubles. Additionally, the Somatic Problems Scale scores provided by the parents correlate overall with the Conduct Problem Scale scores, a fact which is in accordance with the correlation often observed in the literature [34] between somatization and antisocial lifestyle. Sadly, adoptees also seemed more likely to consider suicide than non-adoptees.

\section{Discussion}

\subsection{Somatizing}

Although not necessarily the variable conveying the most powerful adoption effect, one striking finding in the thematic analysis was the high prevalence of chronic somatic troubles among adoptees (Table 4) further confirmed by the comparison of CBCL DSM Somatic Problems Oriented Scales within adoption and control groups (Table 6). What is striking about this is that in the best case, it signals a high-cost solution against a disorganization threat (e.g. through the transitional time/space it provides for the symbolization of a disruptive event) [50], while in the worst case it signals the (renewed) experience of a (past) state of agony (e.g. the adolescent feeling like dying when sick - a characteristic feature of the study) once all defenses against the return of the split off have been overwhelmed/abandoned. Although the somatic problems scores of both parents and the adolescent correlate (moderately) with the presence of somatic troubles identified in the interviews $(r=0.52 ; p=0.000$ and $r=0.31 ; p=0.038$ respectively), parents seemed to underrate such troubles. The mean somatic problems score of adolescents seemed consistent with the higher perceived somatic distress among the adoptees and might illustrate the psychoanalytical concept of state of agony correlated to primary trauma. Possible explanations from a psychoanalytical perspective of why the parents underrated somatic troubles compared to the adolescents - and compared to the results of the thematic analysis could be (1) the presence of a social desirability bias supported by a secondary thought process more present in the adult than in the (pre)adolescent, (2) an underlying denial of the suffering endured by the adolescent, because it either damages the parents'

\footnotetext{
${ }^{3}$ Zero cells ( $\left.0.0 \%\right)$ have expected count less than 5 . The minimum expected count is 5.50 .
}

illusion of being all-powerful [51] or threatens areas which are traumatic for the parents themselves, or (3) both. The theoretical link established by the literature review makes it tempting to correlate somatizing to a lower degree of alpha functioning and of primary symbolization [52], which could be estimated by the ratio between signs of alpha functioning (e.g. creative games and activities, success in achieving own goals, capacity to be alone, drive for acquiring knowledge, capacity to manage time, etc.) and alpha dysfunction on a larger sample.

\subsection{Degree of alpha functioning}

Three aspects of the failing function which emerged during the stage-by-stage thematic analysis were a preoccupation with the presence/absence of other family members, temporality and weak transitional barrier. One adoptee vividly complained that her parents did not leave her enough (internal) objects to occupy their absence, suggesting that the hallucinatory thinking by that adolescent was deprived of the necessary internal objects. Internalizing (of the alpha function) seemed to be a process that adoptees had particularly difficulties with (at least more than non-adoptees seemed to have on average). Meanwhile, the attribution of exaggerated importance to pets (Table 6) seemed to play a huge calming role and therefore to favor internalizing of the alpha function, especially among the youngest adolescents. In such circumstances, the pet may provide the function of protective shield, but also that of object presenting (rejecting, retrieving). Although this is a rather demanding task for a pet, it may be the only one with the ability to perform it, and this makes it precious.

\subsection{Narcissistic identity pathologies}

One way to map the categories of anxiety and defense mechanisms which emerged from the thematic analysis (Table 4) and presence of narcissistic identity troubles among adolescents (Table 1) was to classify categories as a function of the increasing level of organization of the defense, and then at each level to:

- identify the possible shadow of the main primary defense mechanism (the primary splitting) [26];

- detect any presence of the three main and additional defense mechanisms which were once put in place by the child to counter the return of the split off primary trauma as well as detect the presence of other solutions for binding excess energy and reducing excitation levels (Table 5).

The categories of anxieties and defense mechanisms (e.g. helplessness and withdrawal) can assume a different theoretical meaning according to the organization of the defensive mechanism (e.g. avoiding others when sad as an amputation of part of the self, or False Self indicated by withdrawal into own little world).

The mapping of the results shows that all the main signs of the presence of narcissistic identity troubles are present Therefore it appears from the categories that emerged from the data-driven 
and their theory-driven mapping with the components of a psychoanalytical model assuming a traumatic origin for narcissistic identity troubles in adolescents that adoptees of the studied group showed a higher prevalence of narcissistic defenses than their non-adopted peers did ( $76 \%$ of adoptees vs. $48 \%$ in the control group).

\subsection{Sensitive periods}

Implicit sensitive developmental periods supported by various theoretical perspectives would deserve a closer look in future studies on larger adoption population samples, in particular:

- the second six months of life and the following months regarding the level of internalizing of the alpha function and its long-lasting effects on somatic risk;

- the age of 5-8y for the perception of differentiation and a first round of the quest for origins among adoptees (e.g. the child realizes that he/she has been adopted at the transition from nursery to primary school [53], leading to moments of profound sadness and malaise, but also specific health problems);

- the age of 14-15y with its emotional reactivity peak [39].

Especially, possible effects of parental mental dimensions on the way in which the infant and later the adolescent negotiates these three sensitive periods might be worth measuring. In the small studied sample $(n=25)$, the 9 adolescents adopted during their second six months of life (5-11 months old at adoption) also showed overall the highest frequency of chronic somatic troubles at adolescence ( $80 \%$ considering the adjusted indicator compared to an average overall of $36 \%$ for the adoptees). This subgroup was therefore a main contributor to the presence of somatic complaints among the group of adoptees. This may mean that signs of primary trauma and somatic troubles are not necessarily congruent, although they both signal an issue with primary symbolization. The current study suggests that international adoption is very likely correlated to primary trauma (even adolescents adopted in their first trimester of life showed the signs in this study to the same extent as their peers adopted at a later stage), to primary splitting defense mechanisms and to later narcissistic identity pathologies with various levels of defense organizing. Very likely, even the less disruptive circumstances of very early adoption can hardly prevent a drastic failure of the maternal reverie, so that a young adoptee has to split off from experienced, representation-less material, driven by the compulsion to repeat. This point aside, narcissistic defense organization preventing the return of the state of agony, is a solution that preserves the adolescent from the disorganization threat, from psychotic disorders and their associated defense mechanisms, anxieties and relationship patterns. Keeping the split off trauma locked away consumes the self. Still, it might be reasonable to assume that internalizing and stabilizing the alpha function - the primary symbolization process enabler - in the presence of an adequate maternal reverie provider (e.g. adoptive parent), can occur in parallel. A privileged period for the (preliminary) acquisition of this function is the second semester of life.
This period may be particularly sensitive for adoptees, and for any child that has experienced primary trauma. How differences between group symbolic contents (culture/language), which are often a feature of international adoption, interfere with or affect the chance for the adopted child to grasp the alpha function from the maternal reverie at hand, is another question that may deserve further consideration. The extent to which an adoptee tends to somatize (measured by the adjusted somatic troubles indicator) may be an indication of the degree of success of the alpha function stabilization in this sensitive period.

\subsection{Methodological limitations}

The review of the literature provided the initial justification for reading the clinical material collected through a developmental paradigm with a different, psychoanalytical, paradigm by the facts that:

- both agree on the key factors that shape early development; - the method chosen for material collection based on semistructured interviews gave the necessary place to the subjectivity of the interviewees.

The paradox of using the individual singularity to reach intra and inter group inferences should however not be overlooked. This point aside, the subjectivity of the researcher, who was working with a single theoretical framework, i.e. the grounded theory approach, has already been mentioned as a potential source of bias, although a deliberate effort was made to challenge that vision with the results provided by other theoretical perspectives, for example neurosciences. Questions could also be raised about internal validity and reliability limitations due to using only one encoder, although these were partially corrected for. A more serious methodological limitation may lie in the fact that both terms of the research question were operationalized with a single theoretical model [26], implicitly validating the existence of the first term from the clinical observation of the second measured at adolescence. Adoptees were assumed de facto to be more likely to have experienced primary trauma, an assumption supported by empirical research which shows that preadoption length of time and severity of caregiving deprivation are two predictors of neurological and age-level motor development delays. From a quantitative perspective, for the measurement of risk of primary trauma could have been better approximated by measuring age at adoption moderated by the country of origin with a constructed ratio. Given the limited set of data, this option was discarded. The adoption factor alone remained, and did in fact prove sufficient to show a main overall effect.

\section{Conclusion}

The primary objective of the current study was to examine severity of early deprivation - assumed to have been experienced preadoption - as a risk of narcissistic identity pathologies in adolescence in the specific context of international adoption. The answer for the studied sample is clearly that there is an effect, 
although only a moderate one. The affective and cognitive reorganizations taking place during adolescence expose adolescents' narcissistic vulnerability. In this context, adoptees showed:

- a higher activation of narcissistic defenses, in particular compliance, rage, control and withdrawal, which can indicate the need to resort to splitting and the existence of a split off part;

- a higher somatization, in comparison with their non-adopted peers.

The prevalence of the more silent physiological solution (somatization) vs the behavioral solution may indicate poor internalization of the alpha function. Further studies are needed in order to understand the interaction effects of the following factors: age at adoption, parents' empathy and capacity to mentalize [54], support from others, sibling configuration, difference in group symbolic contents which are often a feature of international adoption, etc.).

Two main theoretical implications emerge from this study:

- existing theoretical models describing the general population's affective and cognitive development and dysfunctions are probably sufficient to cover and understand the population of adolescents adopted in infancy;

- the model proposed by Roussillon was particularly useful in correlating recurrent defense organizations with forms of narcissistic identity pathologies and the higher risk of suicide this population incurs.

To the question: "Are adoptees a population with specific issues requiring specific theoretical work?" the answer is therefore no. However, this research suggests that, in addition to their higher risk of suicide, special attention needs to be paid to the higher level of somatic complaints. The higher somatic risk that oscillates within the studied population between self-defense, self-treatment and the experience of a state of agony, probably signals the recurrent failure of the primary symbolization processes, both secondary and primary, suggesting two possible areas of work. One is preventive, the other is therapeutic. The therapeutic perspective suggests that all therapies focusing on the repair or completion of a problematic primary symbolization process are well worth exploring and using with these adolescents. With respect to prevention, stress should be placed on ways to decrease the somatic risk and, as a corollary, to favor the internalizing by the child of the alpha function. In this respect, although adoption as early as possible may appear to be the best option in order to minimize the long-lasting effects of caregiving deprivation observed here, larger studies measuring the effect of sensitive periods in late somatic risk (and internalizing of the alpha function) could contradict such a hasty conclusion.

\section{Disclosure of interest}

The authors declare that they have no conflicts of interest concerning this article.

\section{References}

[1] Brusset B. L'avant-coup trouvé/créé. Rev Fr Psychanal 2009:1539-43.

[2] Green A. Les cas limite. De la folie privée aux pulsions de destruction et de mort. Rev Fr Psychanal 2011:375-90.

[3] Jeammet P. Le développement de l'individu: une co-construction permanente à la merci des rencontres. RFAS 2013:11-4.

[4] Kernberg OF. Quelques observations sur le processus de deuil. Année Psychanal Intern 2011:153-75.

[5] Widlöcher D. Stupeur et figures dans la dépression. In: Dépression du bébé, dépression de l'adolescent. Eres: Toulouse; 2010. p. 13-27.

[6] Kim WJ. Benefits and risks of intercountry adoption. Lancet 2002:423-4.

[7] Brand AE, Brinich PM. Bahavior Problems and Mental Health Problems in Adopted, Foster and Non-adopted Children. J Child Pyschol Psychiatry 1999:1221-9.

[8] Gagnon-Oosterwaa N, et al. Pre-adoption adversity, maternal stress, and behavior problems at school-age in international adoptees. J Appl Dev Psychol 2012:236-42

[9] Palacios J, Moreno C, Román M. Social competence in internationally adopted and institutionalized children. Early Child Res Q 2013:357-65.

[10] Roeber BJ, et al. Gross motor development in children adopted from orphanage settings. Dev Med Child Neurol 2012:527-31.

[11] Wiik KL, et al. Behavioral and emotional symptoms of postinstitutionalized children in middle childhood. J Child Psychol Psychiatry 2011:56-63.

[12] Hjern A, Lindblad F, Bo V. Suicide, psychiatric illness, and social maladjustment in intercountry adoptees in Sweden: a cohort study. Lancet 2002:443-7.

[13] Laubjerg M, Petersson B. Juvenile delinquency and psychiatric contact among adoptees compared to non-adoptees in Denmark: A nationwide register-based comparative study. Nord J Psychiatry 2011:365-72.

[14] Drieu D, Johnston G. Résonances traumatiques familiales chez des adolescents adoptés venant d'une autre culture. Dialogue 2007:45-56.

[15] Cohen NJ, et al. Children adopted from China: a prospective study of their growth and development. J Child Psychol Psychiatry 2008:458-68.

[16] Roskam I, et al. Another way of thinking about ADHD: the predictive role of early attachment. Soc Psych Psych Epid 2013:133-44.

[17] Rutter M, et al. Early adolescent outcomes of institutionally deprived and non-deprived adoptees. III. Quasi-autism. J Child Psychol Psychiatry 2007:1200-7.

[18] Smyke AT. Development and institutional care. Dev Med Child Neurol 2012:487.

[19] Julian MM. Age at Adoption from Institutional Care as a Window into the Lasting Effects of Early Experiences. Clin Child Fam Psychol Rev 2013:101-45

[20] Guedeney N, Dubucq-Green C. Adoption, les apports de la théorie de l'attachement. Enfances \& Psy 2005:4-94.

[21] Anzieu-Premmereur C. Le jeu dans les thérapies parents-bébés. Rev Fr Psychanal 2004:143-55.

[22] Duparc F. Winnicott et la création humaine. In: Winnicott et la création humaine. Toulouse: Eres; 2012. p. 219-39.

[23] Grinberg L, Sor D, Tabak de Bianchedi E. Nouvelle introduction à la pensée de Bion. Lyon: Cesura Lyon; 2006.

[24] Klein M. Les stades précoces du conflit œdipien. In: Éssais de psychanalyse, 1921-1945. Paris: Payot; 1928. p. 229.

[25] Ciccone A. Psychopathologie du bébé, de l'enfant et de l'adolescent. In: Manuel de psychologie et de psychopathologie clinique générale. Masson; 2007.

[26] Roussillon R. Primitive agony and symbolization. London: Karnac Books; 2010.

[27] Roussillon R. Agonie, clivage et symbolisation. Paris: Presses Universitaires de France; 1999

[28] Fagan M. Relational trauma and its impact on late-adopted children. J Child Psychother 2011:129-46.

[29] Harf A, et al. L'enfant adopté à l'étranger, entre langue maternelle et langue d'adoption. La psychiatrie de l'enfant 2012:315-38.

[30] Field T, Diego M, Hernandez-Reif M. Prenatal depression effects on the fetus and newborn: a review. Infant Behav Dev 2006:445-55. 
[31] Fonagy P, Gergely G, Target M. The parent-infant dyad and the construction of the subjective self. J Child Psychol Psychiatry 2007:288-328.

[32] Mascaro R, et al. Evaluation des effets du placement précoce du bébé en pouponnière. Devenir 2012:69-115.

[33] Schore AN. The effects of early relational trauma on right brain development, affect regulation and infant mental health. Infant Mental Health J 2001:201-69.

[34] Lilienfeld SO, Hess TH. Psychopathic personality traits and somatization: sex differences and the mediating role of negative emotionality. J Psychopathol Behav Assess 2001

[35] Dayan J, Guillery-Girard B. Conduites adolescentes et développement cérébral: psychanalyse et neurosciences. Rev Adolesc 2011:479515.

[36] McRae K, et al. The development of emotion regulation: an fMRI study of cognitive reappraisal in children, adolescents and young adults. Soc Cogn Affect Neurosci 2012:11-22.

[37] Stortelder F, Ploegmakers-Burg M. Adolescence and the reorganization of infant development: a neuro-psychoanalytic model. J Am Acad Psychoanal Dyn Psychiatry 2011:203-532.

[38] Lamotte F, et al. Les achoppements de la construction identitaire dans les adoptions internationales. Neuropsychiatr Enfance Adolesc 2007: $381-8$

[39] Pfeifer JH, Blakemore SJ. Adolescent social cognitive and affective neuroscience: past, present, and future. Soc Cogn Affect Neurosci 2012: $1-10$.

[40] Strauss A, Corbin JM. Basics of qualitative research: grounded theory procedures and techniques. Thousand Oaks, CA, US: Sage Publications; 1990 [Inc. 270].

[41] Aber JL, et al. The parent development interview. 1985.

[42] Steele M. A longitudinal study of previously maltreated children: attachment representations, adoption, in developmental science, psychoanalysis: integration, innovation, Y.C., s., Center, Editor. 2003: New Haven, CT.
[43] Achenbach TM, Rescorla LA. Manual for the ASEBA school-age forms and profiles. Burlington: University of Vermont, research center for children, youth, and families; 2001.

[44] Halfon O. Adopted adolescents: attachment and behavior problems "Core project" submitted to the teams of the network "adoption/attachment/adolescence". Lausane: Lausanne University Child and Adolescent Psychiatry Dept; 2009

[45] Stievenart M, et al. Friends and family interview: measurement invariance across Belgium and Romania. Eur J Dev Psychol 2012:737-43.

[46] Voynnet Fourboul C. Ce que «analyse de données qualitatives » veut dire. Revue internationale de psychosociologie et de gestion des comportements 2012:71-88.

[47] Kohut H. Observations on the psychological functions of music. J Am Psychoanal Assoc 1957:389-407.

[48] Kreisler L. La psychosomatique de l'enfant. $4^{e}$ Éd. Paris: Presses Universitaires de France, coll. "Que sais-je"; 1992.

[49] Achenbach TM, Dumenci L, Rescorla LA. DSM-oriented and empirically based approaches to constructing scales from the same item pools. J Clin Child Adolesc Psychol 2003:328-40.

[50] Debray R. Expression somatique et transitionnalité. Rev Fr Psychanal 1995:1571-3.

[51] Colbère MT. Adoption et adolescence : parents en question(s). Dialogue 2001:89-98.

[52] Fine A. Interrogations sur le défaut de symbolisation primaire comme paramètre du phénomène psychosomatique. In: Métapsychologie : écoute et transitionnalité. Paris: Presses universitaires de France; 1995. p. 1699-704.

[53] Bebiroglu N, Pinderhughes EE. Mothers raising daughters: new complexities in cultural socialization for children adopted from China. Adoption Quarterly 2012:116-39.

[54] Debray R, Belot RA. La psychosomatique du bébé. Paris: Presses Universitaires de France; 2008. 